\title{
Virtual Reality (VR) Multi-User Lab for Immersive Teaching
}

Savickaite, S., Millington, E., Latkovskis, I., Failes, J., Kirkwood, N., and McDonnell, N.

\begin{abstract}
As VR technology matures, it offers opportunities to provide state of the art learning experiences. The use of this technology in education is not new, however, it can be significantly improved. Situated (or contextual) learning is one of the key pillars of immersive learning. Multi-user interaction in virtual environments has always been one of the goals of VR and this has been represented by the tools developed for it. In this preliminary work, we aim to introduce Project Mobius, which is a collaborative project between Edify.ac and the University of Glasgow. We describe how our multiuser lab has been set up and potential future applications for teaching and learning.
\end{abstract}

Key words: virtual reality, multi-user, immersive technology, teaching 


\section{Introduction}

Virtual reality (VR) is as hard to define as the experience itself. VR systems are a sophisticated interplay of technology and human perception, most commonly associated with Head Mounted Displays (HMDs), which use a display in front of each eye to create an illusion of immersive threedimensional space around the user. VR systems can also incorporate several input devices, such as controllers or gloves.

Virtual Reality (VR) has been used in research since the late 1980s, however, since the milestone acquisition of Oculus by Facebook in 2014 (Polcar \& Horejsi, 2013), VR has come into focus as an available and useful tool for research. Equipment has become cheaper, more advanced, and more available to all. VR now offers authenticity and a high level of realism as well as experimental control, thus facilitating learning and transfer of skills in the real world.

As VR technology matures, it offers opportunities to provide state of the art learning experiences. The use of this technology in education is not new, however, it can be significantly improved (Herold, 2016). Situated (or contextual) learning is one of the key pillars of immersive learning (Harrington, 2006). VR environments provide realistic scenarios and allow the learning of concepts that might be too abstract to explain in only two dimensions, or learning in dangerous (or inaccessible) environments, such as on top of a volcano or inside a human brain. Several studies have found VR immersive learning beneficial (Parong \& Mayer, 2018; Lin et al, 2019; Makransky et al, 2019), however, the phenomenon of immersive learning needs to be explored further and different learning strategies disseminated.

\section{Collaborative multi-user VR}

Multi-user interaction in virtual environments has always been one of the goals of VR and this has been represented by the tools developed for it. AltspaceVR is just one example of many offering an interactive social space in VR. Education is then an obvious extension of these tools. Collaborative learning in virtual worlds has been extensively researched (Greenwald et al, 2017; Slater, 2017; Dede et al, 2017), however, these studies are limited to users joining the same virtual simulation, generally joining from multiple, separate locations. To our knowledge, nobody has investigated a multi-user VR room, where users are engaged in the same virtual learning activity, but share a physical space with multiple headsets. Multi-user VR spaces are quite popular in VR arcades, but the concept has not yet been widely adapted for educational purposes.

In this preliminary work, we aim to introduce Project Mobius, which is a collaborative project between Edify.ac and the University of Glasgow. We describe how our multi-user lab has been set up and potential future applications for teaching and learning.

\section{Equipment}

We set up 15 VR bays for students to use. Each of the 15 bays was fitted with HTC Vive Pro HMDs connected to a workstation powered by a Nvidia Quadro P5000 graphics card. Each workstation was fitted with a touchscreen monitor so that their user's view within VR could be externally observed. Eventually, the HTC Vive Pro kits were replaced with HTC Vive Focus 3 kits for reasons which we will go on to discuss.

As the COVID-19 pandemic was evolving, it became clear that sufficient hygiene precautions would have to be taken for the multi-user VR Lab. For this reason, we fitted the lab with 8 ultra-violet HMD cleaning kits manufactured by CleanBox. These kits use a segment of ultra-violet light known as UV$\mathrm{C}$ to eliminate all bacteria and viruses on the headset and handheld controllers without damaging the devices. 


\section{Permanent classroom}

A permanent classroom was established at the Partick Burgh Hall in Glasgow. The room's dimensions were $12 \times 12 \mathrm{~m}$. The 15 identical 'Play Areas' were set up. Due to the size of the room, 12 base stations were placed around the space to ensure optimal tracking of all users (Figure 4).
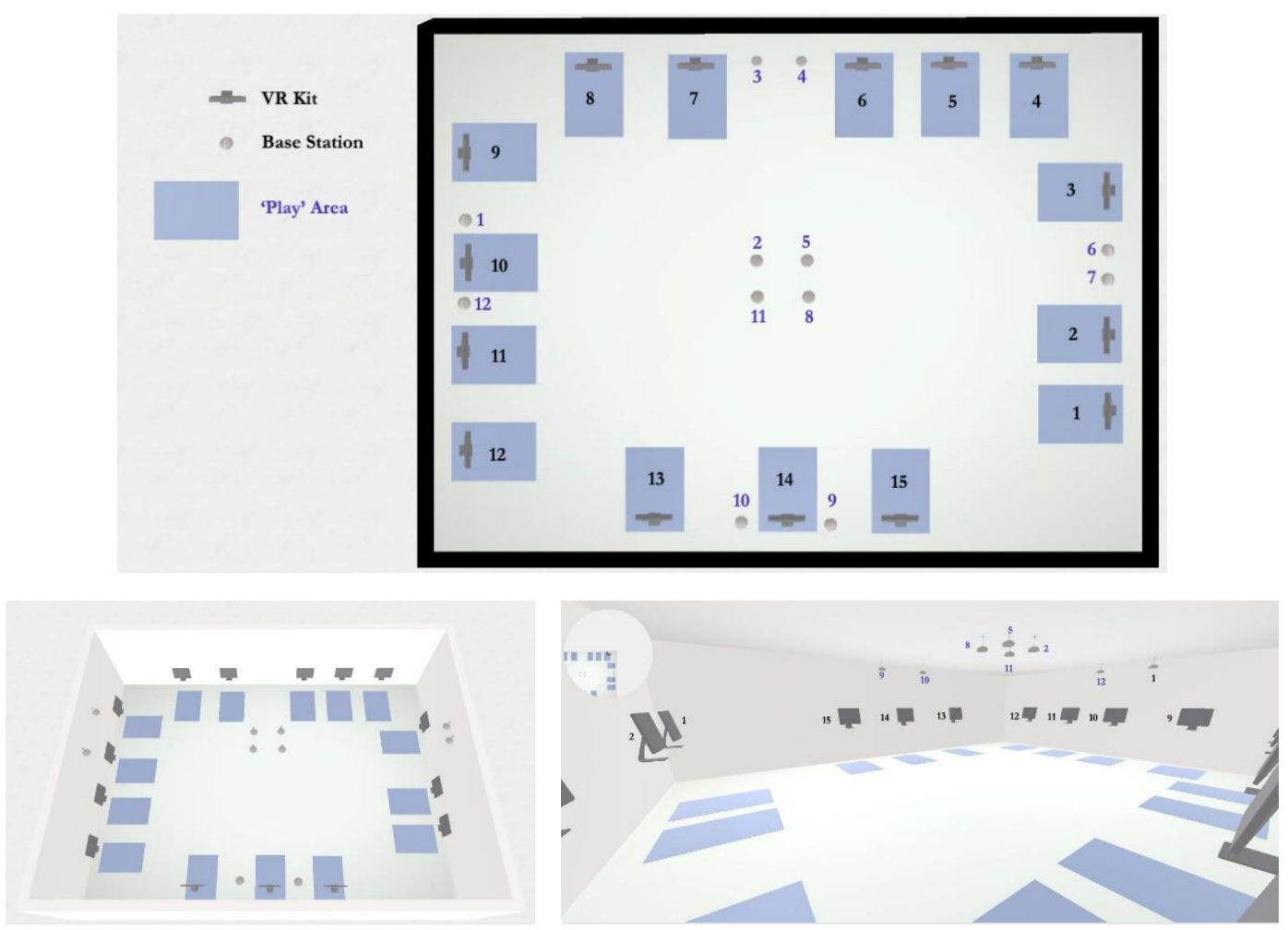

Figure 1. Partick Borough Hall multi-user VR lab set up.

As previously mentioned, the stationary lab initially used HTC Vive Pro kits. These headsets track their movement in space using external base stations which emit infrared light. However, as the project progressed, HMDs which track movement using inbuilt optical cameras, known as inside-out tracking, became more available on the consumer market. While this kind of tracking has certain limitations (e.g., the headset may lose tracking of handheld controllers if they are moved outside of the tracking angle), it nonetheless avoids a number of problems in setting up a multi-user lab, such as configuring communication between base stations. For this reason, we opted to replace the HTC Vive Pro kits with HTC Vive Focus 3 kits, which feature inside-out tracking.

By replacing the headsets, we were able to dismantle the complicated base station tracking system. Each headset was connected to a workstation using a proprietary USB streaming cable. The change of headsets made little difference to the end-user as they would still be wearing an HMD connected to a $\mathrm{PC}$ via a cable.

Trip hazards were identified as a key risk when the 'Play Areas' were being set up. Opting for a wireless headset can reduce the risk of tripping over the wires. To reduce the trip risk, we installed retractable cables overhead, affixed with loops, which held the streaming cable in place (away from the users' feet) and allowed each user to traverse their play area comfortably and without the risk of the cable tripping them up. 


\section{Pop-up Lab}

A second lab was installed in a student union event space. The guiding idea behind this lab was that it would be a 'pop-up' space - requiring little permanent infrastructure - where headsets could be stored away in a cupboard when not in use. We opted to populate this lab with Oculus Quest and Oculus Quest 2 headsets. Unlike the headsets used in Partick Burgh Hall, the Quest headsets do not require a connection to a PC. They are powered entirely by lightweight mobile-phone processors built into each headset. As a consequence, such headsets have less computational power and can only run VR apps that have been significantly scaled down from their PC-VR counterparts. The advantage of such a setup is that a VR experience does not require bulky fixed infrastructure in the form of PCs, monitors and cables.

A standalone VR lab also does not require permanent, physically marked-out play spaces. Each user starts their VR experience with the prompt of drawing out their play space. This is made possible with the use of in-built optical cameras which allow each user a 'pass-through' view of the world around them. Users then use their handheld controllers to mark a perimeter around them. If at any point the user steps out of the perimeter they have drawn for themselves, the pass-through cameras will override the VR view, thereby telling the user to return to their play space if they wish to continue their VR experience.

This lab was also fitted with 16 UV Cleanboxes which would be used to clean and disinfect the headsets between uses.

An ongoing challenge for this lab is the development of VR applications that will function on standalone HMDs. Currently, the Edify suite of apps is designed for PC-VR, so adapting them to standalone VR will incur significant development time and may potentially result in a worse visual quality resulting in poor model fidelity. Thus, it might need to be scaled back on graphical complexity.

It is also the case that in the absence of a direct feed to a PC monitor, it is more difficult for a teacher or tutor to offer guidance to students who are having difficulty. To address this important aspect of VR lab teaching, we have installed two high-end wireless access points (Cisco 9130) and four Chromecast devices connected to existing TV screens. With this infrastructure in place, up to four students at a time can cast their experience from the headset to the TV screens for the teacher to see. User literacy of this function should be ensured before the headset is worn.

\section{Individual VR pods}

Finally, a single-user VR lab was set up in an office at the University of Glasgow, dubbed an 'Edify Pod'. Several other Edify pods are planned to be established in the future. The idea behind this lab was to give teaching staff a room they can book which would have all the necessary tools for developing their own immersive lessons through the Edify Lesson Creator application. These lessons could then be delivered to students in one of the teaching labs.

The inaugural Edify pod was fitted with an HTC Vive Pro kit, complete with base stations installed in the corners of the room to allow full tracking. This was connected to a workstation powered by a GTX 1080 graphics card. A UV-C Cleanbox was also installed here.

\section{Project Mobius exemplar lessons}

Project Mobius created ten VR applications, each of which enables teaching that would not be possible without immersive technology. These apps span across multiple disciplines and were selected from entries to a VR Teaching Competition held at the University of Glasgow in 2017. Geology, Data Science, Anatomy, Immunology, Philosophy, History, Physics, Chemistry and Physiology are all 
represented in this initial tranche of apps - see acknowledgements below for a list of contributing staff.

1. Field course of Arran. Students can 'visit' a geological location on the Isle of Arran in Scotland in VR. Students can undertake measurements of geological features and their characteristics, record and repeat these, and view the whole geological feature from a bird's eye view.

2. Introductory Physics - Projectile Motion. This app provides students with a flexible and immersive VR Physics laboratory, allowing them to perform a "ball bouncing" experiment while manipulating gravity, friction and other parameters.

3. Mutations on Protein Molecular Structure. Molecules from the extensive online Protein Data Bank (The Protein Data Bank) can be uploaded and viewed in 3D space, enabling students to understand protein structures, measure distances between amino acids and compare genetic mutations.

4. Seeing Data Landscapes. This app is a data visualisation tool, enabling lecturers and students to intuitively explore and manipulate three-dimensional data from a first-person perspective.

5. Printing Press. Users are taken back to the origins of book printing. They are guided through the operation of the Gutenberg Press, from typesetting and inking to operating the press by hand.

6. Digestion. Students are taken to the stomach, where they can observe the digestive process, control a simulation of a variety of cells, and follow their effects at both the macro- and microscopic levels.

7. Battling Infection. Users follow a patient's battle with salmonella and control their immune response. The app follows the disease's course through the intestinal tract, submucosa and lymph nodes.

8. Cardiovascular Anatomy and Physiology. Students are provided with a detailed breakdown of the structure of the heart alongside a 3D model. They then spend time within a blood vessel, controlling the administration of various hypertension medications, and learning about their effects.

9. Trolly Problem. This app visualises a popular philosophical problem by placing users in control of an emergency situation where they must choose to either stand idle and allow a group of workers to be killed by a train, or to actively change the track to allow a bystander on the other track hit instead.

10. Disease Diagnostics Lab. Students are given access to a replica disease diagnostics lab space, where they run a qPCR experiment on the Zika virus, learning to handle pipettes and the associated equipment.
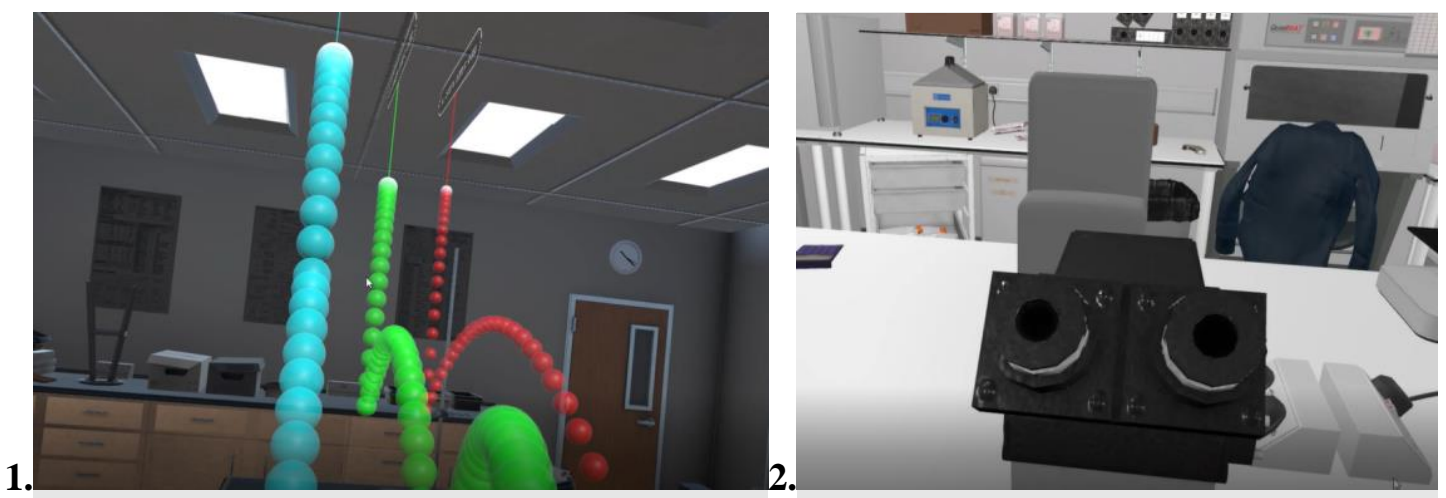

Figure 1. Still images from Project Mobius apps: 1. Introductory Physics; 2. Disease Diagnostics. 


\section{Preliminary User Testing}

Overall, our users were engaged with the virtual environments. We found that users were willing to freely explore virtual environments outside of the tasks set out for them. For example, whilst exploring the environment in the Disease Diagnostics app, one user was keen to look through the microscope. Perhaps due to the immersion, the user was surprised that it did not function as a real-life microscope. The user felt more at ease and comfortable navigating this app more, in comparison to the Printing Press app. It can be due to simple adjustment to the VR environment and the controllers, or psychological familiarity. Although the user was not a scientist, many objects around the lab were more familiar than the medieval atmosphere of the Printing Press. As a result, perhaps this increased the confidence in the navigation. This psychological effect should be explored further. Alfonso \& Beckhaus (2011) discussed a similar effect people tend to be more willing to manipulate objects in a virtual world in comparison to the real world. There is, however, a lack of studies around the behaviour around familiar and unfamiliar environments/objects, and how they affect participants interactions in the virtual world.

Instructions were very clear and easy to follow in all of the apps tested. Controllers were used correctly with only minor errors. There was some confusion around the two stops of the pipette in the Disease Diagnostics app, which should not be an issue if somebody familiar with chemistry is using the app. However, if it is intended to train the beginners some description of the relevance of the two steps of the pipette would be helpful.

The user commented on the text-heavy instructions at the start in most of the apps, particularly in the Introductory Physics app. As a dyslexic adult one user found it difficult to read such a large paragraph in VR. Text and fine detail perception have been found to be difficult in VR (Gerschutz et al, 2019), however, it has not been thoroughly explored in the neurodivergent population. Our user suggested breaking down the instructions into separate smaller paragraphs, where you could click next after reading each part of the instructions. Around 2.6\% of students in Higher Education and 2.28\% in schools (HESA figures from 2005/6) have diagnosed dyslexia. The proportions of other learning disabilities vary, but there is a clear need to discuss how these apps are perceived by neurodivergent users. Some basic psychology reviews are available on how neurodiverse individuals interact with VR (Boyd, et al, 2018; Lopez-Carral et al, 2022; Thomsen \& Adjorlo, 2021).

Very good feedback was received on the quality of the graphics and the immersion. Some issues with adjusting to the controllers were noted, but these improved with practice.

\section{Data extraction and analysis}

The data collected by the Head Mounted Displays are varied and rich, especially when compared to assessment sheets. However, this data can be overwhelming and needs to be processed to be used effectively.

The shift towards online learning as a result of isolation and distancing protocols has demonstrated some of the capabilities of technology to provide intuitive information on how students are interacting with lesson materials, as well as their formal submissions. Examples include whether the student has remained on a lesson page, how long they spent on each question, and the results of their automatic feedback. The data analysis demonstrated in this report is an extension of this style of data reporting.

The initial unique data which is available is the student's position in physical and virtual space. The following examples were collected in the Disease Diagnostics lesson. User interaction data from the Mobius apps can be exported as a .json file and converted to .csv format for further analysis. An example of how this data can be visualised is presented in Figures 2 and 3. 
The interpretation of this information depends on the task the student is completing. In this case, we can see that the student was stationary in front of the Laminar flow hood, using their right hand to interact with the environment. Based on this information, we can assume that the student was focused on the task in front of them, rather than moving to explore the scene. Their left hand was also underutilised, which could be a teaching point for the future.
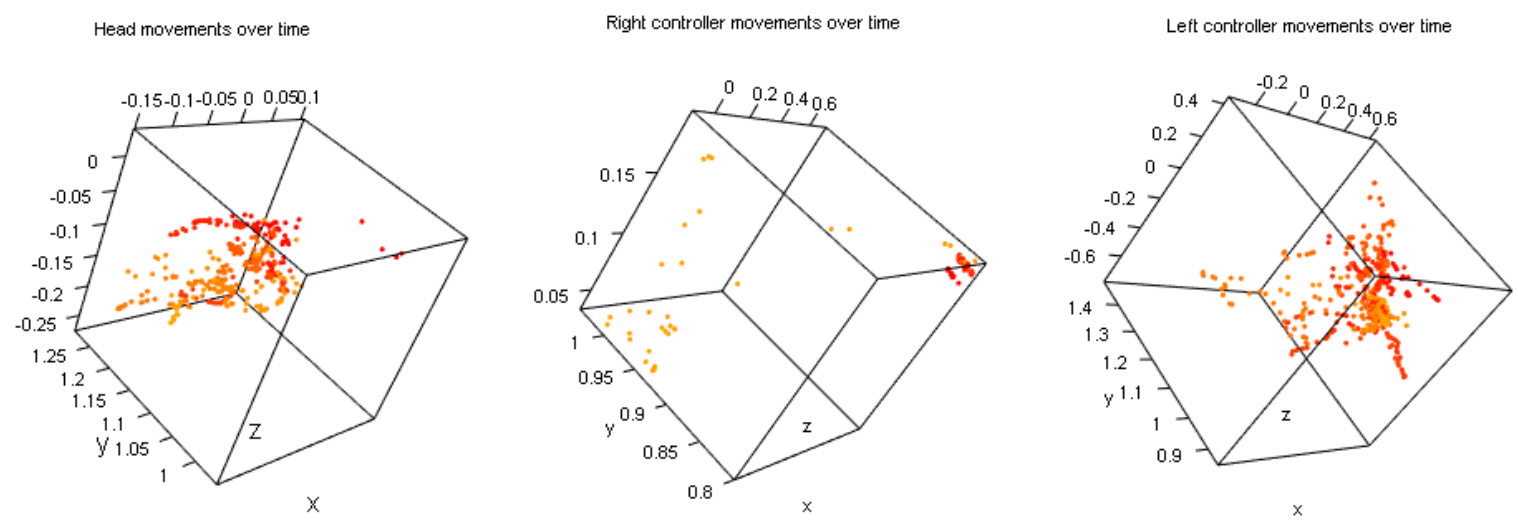

Figure 2. Example of how coordinates of head and controllers' movements can be visualised using R.

\section{Student 3D Position}

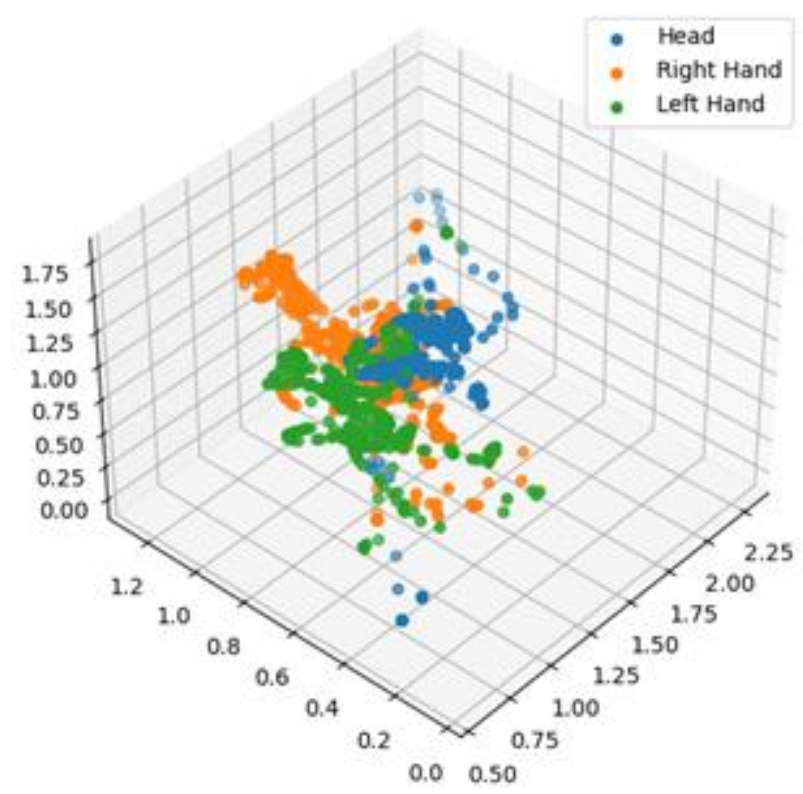

Figure 3. Example of how head and controller coordinates can be represented in a single 3D space.

Based on the data currently being outputted, it is also possible to replicate what the student was doing at any given moment. This could be useful to gain a more intuitive understanding of the other outputs or to compare different students based on an interesting event or timestamp.

As we have seen here, it is possible to gain good qualitative insights into a student's engagement with a lesson using a small slice of the information that can be collected using the edify platform and the Mobius lessons. However, it is also possible to derive improvements for a teacher's virtual reality 
lesson by aggregating together the data from a class of students. For example, teachers can use the positional data to work out whether students are spending appropriate amounts of time at a certain station. If not the difficulty of the task may be too high, or students may be missing visual cues.

\section{Conclusions and outputs}

The next stage of Project Mobius will be to develop the underlying platform that can support such a diverse range of applications and is fit for implementation in higher education. The platform will allow for the development and integration of further apps in the future and will crucially support the capture of anonymised learner data.

Project Mobius will eventually deliver an unprecedented level of data about how learners learn. It will do this by teaching appropriate topics in VR, and passively collecting data about how users behave and learn whilst they participate. Crucially, this is anonymised data - we want to know about learning in general, but we are determined to protect individual students' privacy as we do. We believe that the detail, and consistency, of the data produced by Project Mobius could help us transform education quite generally - be it through immersive, or traditional, means.

\section{References}

Dede, C., Grotzer, T. A., Kamarainen, A., \& Metcalf, S. (2017). EcoXPT: Designing for deeper learning through experimentation in an immersive virtual ecosystem. Journal of Educational Technology \& Society, 20(4), 166-178.

Dictionary, M. W. (2002). Merriam-webster. On-line at http://www. mw. com/home. htm, 8.

Greenwald, S. W., Kulik, A., Kunert, A., Beck, S., Fröhlich, B., Cobb, S., ... \& Maes, P. (2017). Technology and applications for collaborative learning in virtual reality. Philadelphia, PA: International Society of the Learning Sciences.

Herold, B. (2016). Technology in education: An overview. Education Week, 20, 129-141.

Lin, Y., Zheng, L., Zheng, Z., Wu, Y., Hu, Z., Yan, C., \& Yang, Y. (2019). Improving person reidentification by attribute and identity learning. Pattern Recognition, 95, 151-161.

Makransky, G., Borre-Gude, S., \& Mayer, R. E. (2019). Motivational and cognitive benefits of training in immersive virtual reality based on multiple assessments. Journal of Computer Assisted Learning, 35(6), 691-707.

Parong, J., \& Mayer, R. E. (2018). Learning science in immersive virtual reality. Journal of Educational Psychology, 110(6), 785.

Polcar, J., \& Horejsi, P. (2013). Knowledge acquisition and cyber sickness: a comparison of VR devices in virtual tours. Science.

Slater, M. (2017). Implicit learning through embodiment in immersive virtual reality. In Virtual, augmented, and mixed realities in education (pp. 19-33). Springer, Singapore.

Afonso, C., \& Beckhaus, S. (2011, September). How to not hit a virtual wall: aural spatial awareness for collision avoidance in virtual environments. In Proceedings of the 6th Audio Mostly Conference: A Conference on Interaction with Sound (pp. 101-108).

Gerschütz, B., Fechter, M., Schleich, B., \& Wartzack, S. (2019, July). A review of requirements and approaches for realistic visual perception in virtual reality. In Proceedings of the Design 
Society: International Conference on Engineering Design (Vol. 1, No. 1, pp. 1893-1902). Cambridge University Press.

Boyd, L. E., Day, K., Stewart, N., Abdo, K., Lamkin, K., \& Linstead, E. (2018). Leveling the playing field: Supporting neurodiversity via virtual realities. Technology \& Innovation, 20(1-2), 105116.

López-Carral, H., Blancas-Muñoz, M., Mura, A., Omedas, P., España-Cumellas, À., Martínez-Bueno, E., ... \& Verschure, P. F. (2022). A Virtual Reality System for the Simulation of Neurodiversity. In Proceedings of Sixth International Congress on Information and Communication Technology (pp. 523-531). Springer, Singapore.

Thomsen, L., \& Adjorlu, A. (2021, March). Designing a collaborative virtual reality system to assess social inclusion among neurodiverse students. In 2021 IEEE Conference on Virtual Reality and 3D User Interfaces Abstracts and Workshops (VRW) (pp. 353-357). IEEE.

The Protein Data Bank H.M. Berman, J. Westbrook, Z. Feng, G. Gilliland, T.N. Bhat, H. Weissig, I.N. Shindyalov, P.E. Bourne (2000) Nucleic Acids Research, 28: 235-242. doi:10.1093/nar/28.1.235 